\title{
Otoplasty: a graduated approach
}

\author{
Ahmed Soliman Ismail(i)
}

\begin{abstract}
Background: Bat ear deformity is a common presentation among the ENT patients (22.5\%). Many surgical procedures have been described to manage such a problem. A thorough study of the anatomical anomalies contributing to such deformity is required by any plastic surgeon in handling this anomaly.

Results: Modified anterior scoring (Chong-Chet) was done on 45 bat ear deformities in 31 patients. Good surgical results were obtained in $97.7 \%$ of patients, and the surgical time ranged from 60 t0 90 min. Postoperative pain and infection were minimal and effectively managed.

Conclusions: The modified Chong-Chet anterior scoring is a reliable and effective surgical tool for managing bat ear deformity done through one postauricular incision. This technique could be used in young as well as adult patients because it renders the auricular cartilage easier to manipulate.
\end{abstract}

Keywords: Otoplasty, Prominent ear, Bat ear

\section{Background}

The prominent ear is a common complaint by the ENT patients with a recorded prevalence of up to $22.5 \%$. Patients with such a deformity may suffer many psychological and social traumas, which are proven to be dramatically improved after Otoplasty [1].

Surgical correction of a prominent ear deformity requires a thorough understanding of the anatomical composition of the auricle. This structure is composed of a fibroelastic cartilage enveloped in its perichondrium. The skin of the auricle is adherent to the perichondrium at the anterior surface while is separated by a layer of loose areolar connective tissue above the posterior perichondrium [2].

The auricle is composed of 5 major anatomical elements; the helix, the antihelix, tragus, the concha, and the ear lobe. Elements of lesser importance are antitragus, triangular fossa, concha cymba, and cavum [2].

Assessment of auricular protrusion is made at three points; the most superior aspect of the rim, the postlateral projection point of the mid-auricle, and the third point is at the level of the inferior helical rim. The

Correspondence: ahm2005@gmail.com

Faculty of Medicine, University of Alexandria, Alexandria, Egypt average measures for such points are $10-12 \mathrm{~mm}, 20-22$ $\mathrm{mm}$, and $16-18 \mathrm{~mm}$, respectively (Fig. 1 ).

Causes of prominent ears include conchal hypertrophy or excess (upper pole, lower pole, or both), inadequate formation of the antihelical fold (the root, superior crus, inferior crus, or all), a conchoscaphal angle greater than $90^{\circ}$, a combination of conchal hypertrophy, and underdeveloped antihelical fold (Fig. 2) [3-5].

The generally accepted goals of otoplasty have been well described and include decreasing the prominence and protrusion of the ear, producing an antihelical fold, and superior crus of absent or effaced and making the lobule proportionate to the rest of the ear [3].

In addition, any surgical techniques must gain a natural look of the auricle avoiding the pinned ear appearance and allow for symmetry. Popular techniques include variations on Masturdè, Furnas, and Stenstrom cartilage scoring techniques [4-8].

\section{Methods}

The forty-five ears in thirty-one patients have been included in the present study. Twenty-four male patients with age varying from 5 to 40 years old, and 9 female patients aging from 6 to 34 years old. 


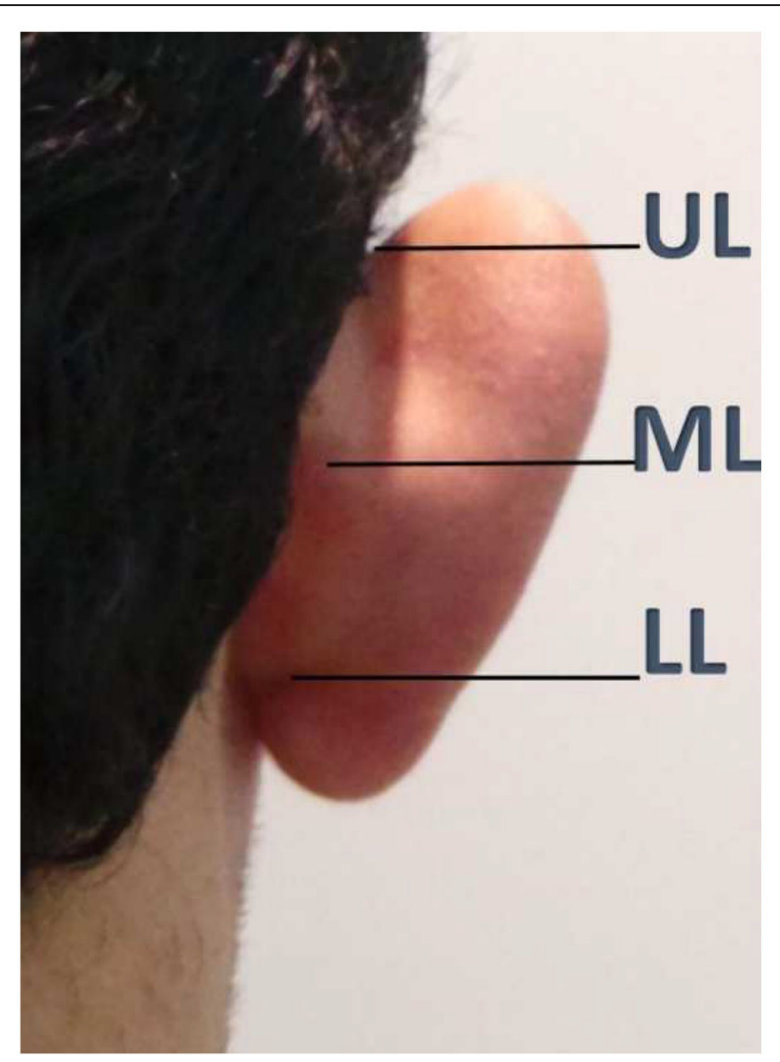

Fig. 1 Demonstration of the three lines marking the auricular protrusion at the UL (upper level), ML (middle level), and LL (lower level)

One surgical procedure was used in all of the study group which is based on the Chong-Chet anterior scoring technique of the auricular cartilage [9].

With the patient in the supine position with the head tilted to the opposite side, the auricle was tilted to the back touching the prominence of the mastoid bone, and marking of the position and thickness of the antihelical fold was done with a marking pen (Fig. 3), using a 25-g needle, piercing the auricular cartilage, then soaking the tip of the needle in a methylene blue solution, and retrieving the needle so the exact line of demarcation is imprinted on the auricular cartilage. An elliptical incision site then was drowned on the postauricular skin extending from the level of the scaphoid fossa to the level of the antitragus, then excision of this skin elliptical graft.

The skin of the postauricular region was then elevated to the level of the start of the helical fold superiorly and lateral from one side and to expose the mastoid region medially.

Using the size 15 scalpel, an incision was made separating the antihelical cartilage from the rest of the auricular cartilage from above downwards.

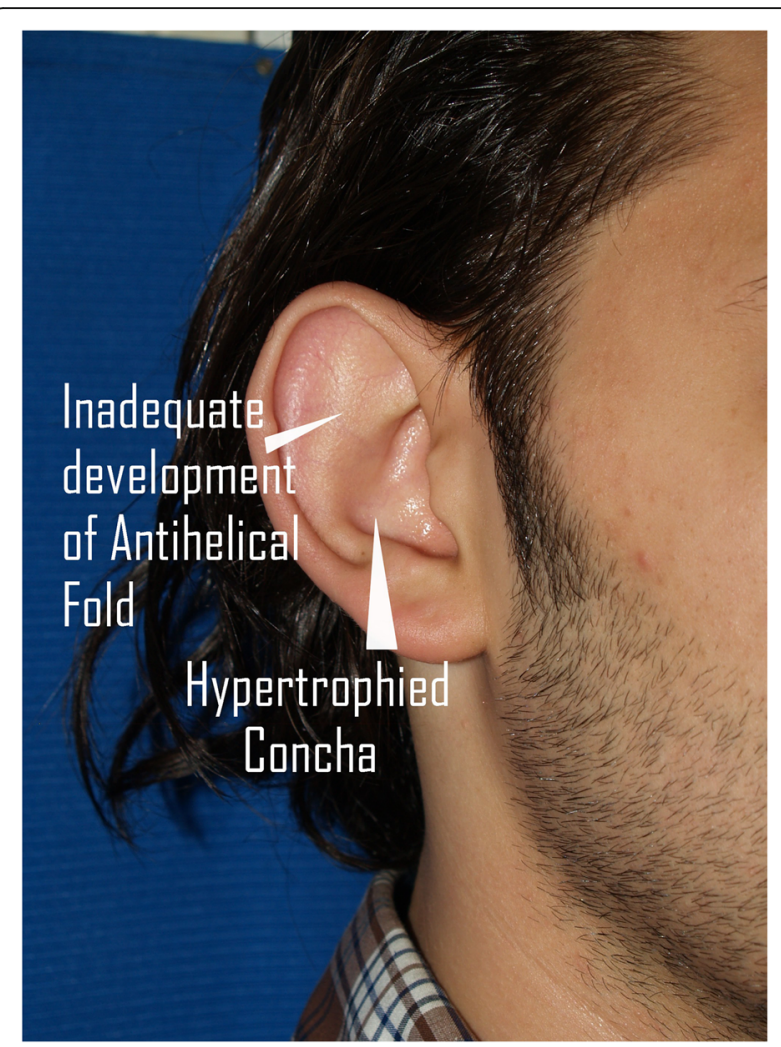

Fig. 2 Right auricle showing 2 of the common causes of auricular protrusion: (1) underdeveloped antihelical fold and (2) hypertrophied conchal cartilage

Elevation of the anterior perichondrium from the auricular cartilage to the level of the conchal cartilage was then done, and hemostasis was achieved using a bipolar forceps (Fig. 4).

Following the site of the mapped antihelical fold, anterior scoring of the auricular cartilage was done using a size 4 soft diamond burr aiming at the weakening of the auricular cartilage and making if malleable at the site of the creation of antihelical folding (Fig. 5).

The antihelical fold was then created using $4 / 0$ polydioxanone sutures (PDS), at the site of preformed antihelix marked by the methylene blue needle points creating the desired fold. Using a 4/0 clear PDS sutures, the approximation of the newly formed concha to the periosteum over the mastoid bone was done in the same technique of the Furnas Technique.

Hemostasis of the surgical field using a Bipolar forceps was done prior to subcutaneous suturing of the surgical wound with 5/0 polyglactin (Vicryl) sutures for the subcutaneous sutures and 5/0 clear polypropylene (Prolene) sutures for the skin (Fig. 6).

Posturicular soft gauze with antibiotic ointment was then placed behind the ear and over the auricular folds then the whole ear was wrapped for a period of 1 week then removed. 


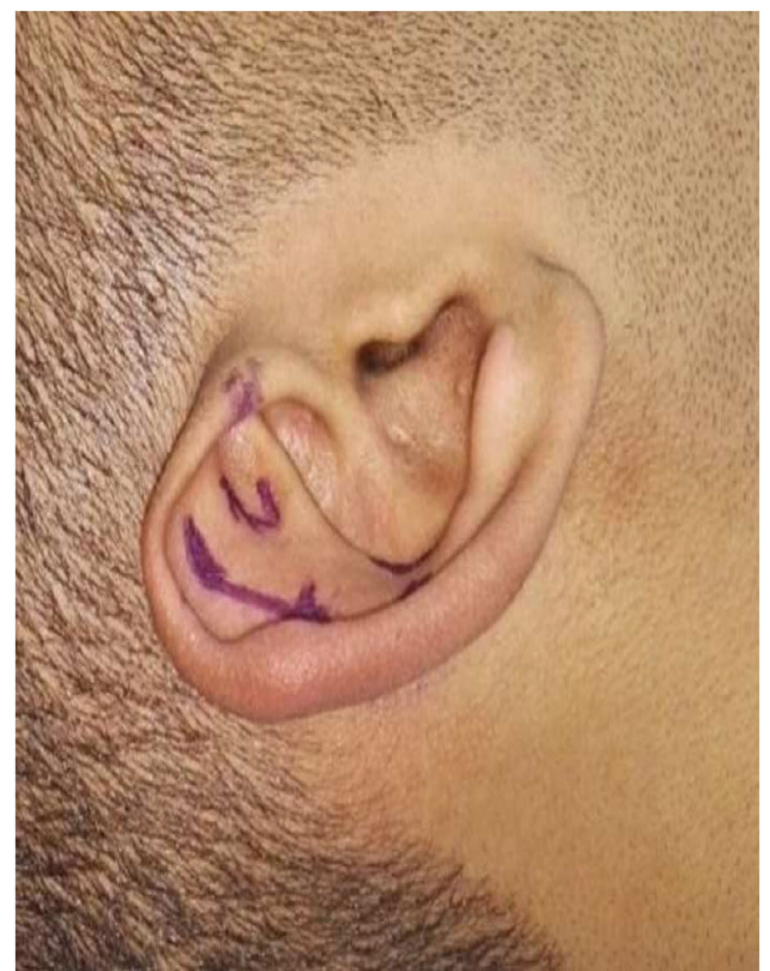

Fig. 3 Right auricular protrusion with the mapping of the supposed site of antihelical fold prior to surgical intervention

\section{Results}

The forty-five ears in thirty-one patients have been included in the present study; 24 male patients with age varying from 5 to 40 years old and 9 female patients aging from 6 to 34 years old.

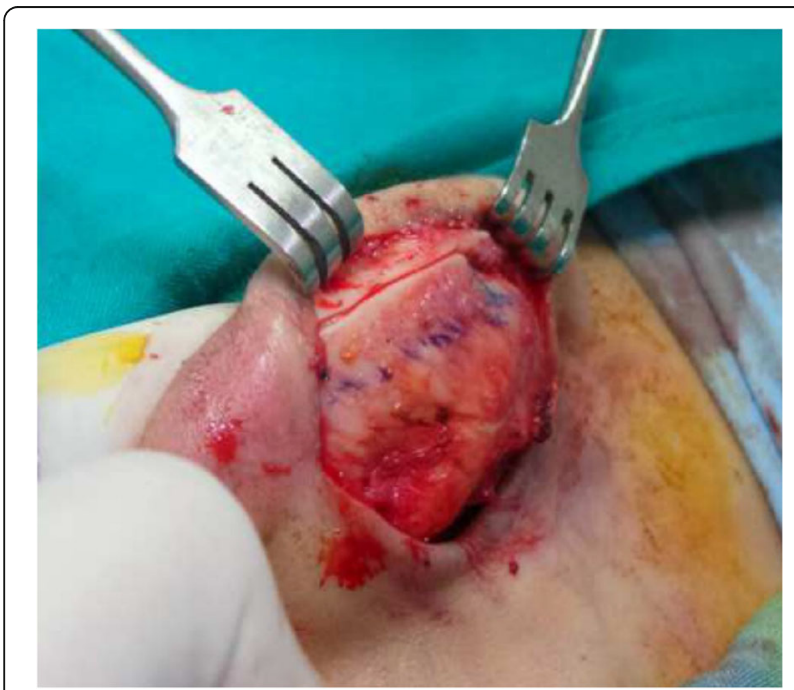

Fig. 4 The site for the creation of the antihelical fold is marked with methylene blue, and the cartilage is separated from the helical cartilage

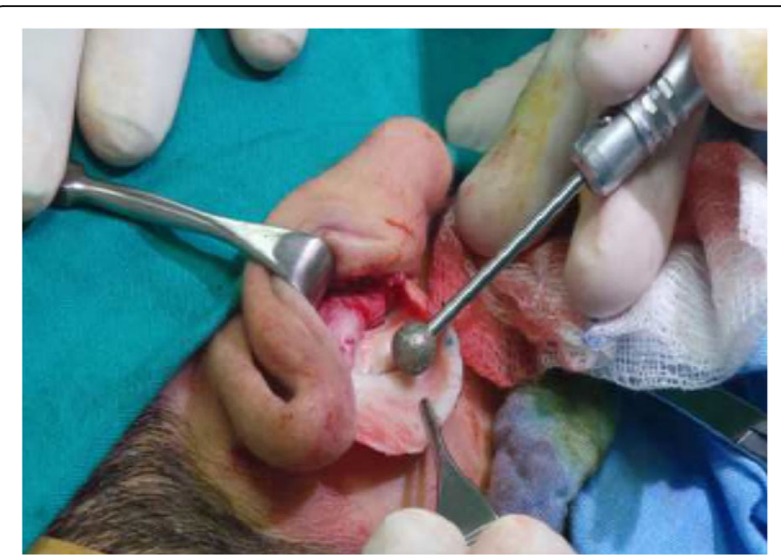

Fig. 5 After elevation of the anterior perichondrium, a diamond burring at the supposed site for the antihelical fold is designed at the weakening of the auricular cartilage and making it more malleable

The surgical time ranged from 60 to 90 min per ear. Postoperative hematoma at the surgical site occurred in one occasion which was dealt with by reopening of the surgical wound and evacuation of the hematoma and hemostasis.

Postoperative pain for a period of 5 days was complained by three cases which were dealt with using antiinflammatory medications. Wound infection after 5 days was encountered in one patient and was dealt with proper antibacterial therapy.

Good results were achieved in 44 out of the 45 ears with one patient (same patient who had hematoma formation) needed a revision surgery 6 months postoperatively (Figs. 7, 8, and 9).

\section{Discussion}

The prominent ear is a common complaint by the ENT patients with a recorded prevalence of up to

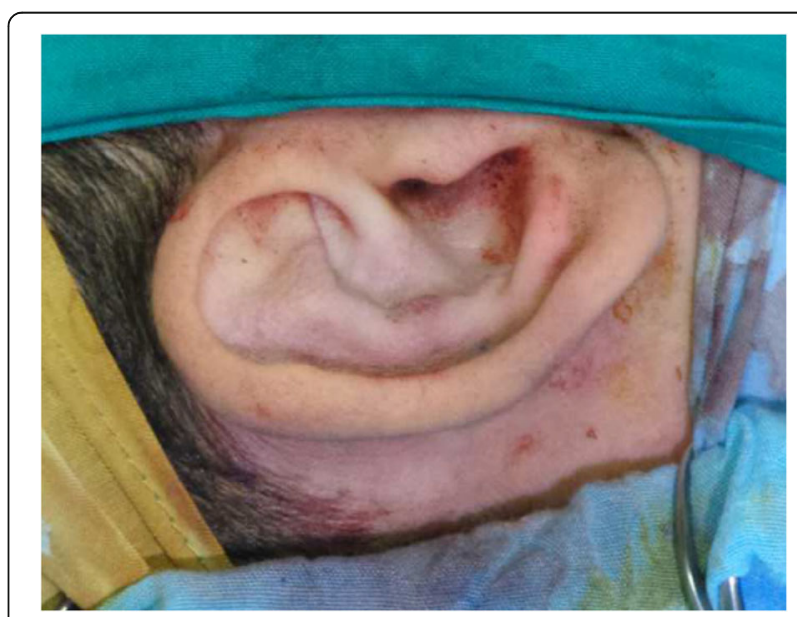

Fig. 6 At the end of the procedure, a well-formed antihelical fold is created 


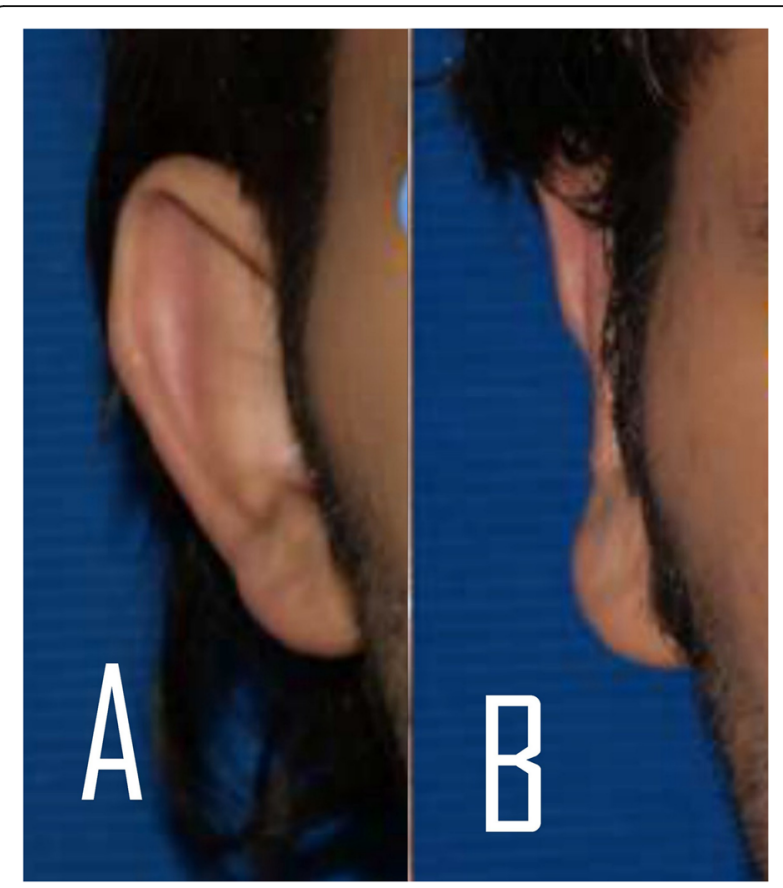

Fig. 7 Preoperative (a) and postoperative (b) shape of the right auricle in a 32-year-old male (frontal view)

$22.5 \%$. Such a deformity may cause many psychological and social traumas to the affected persons. These traumas are proven to be dramatically improved after otoplasty [1].

Surgical correction of the prominent ear was described by many facial plastic surgeons with the aim of decreasing the causing factors such as hypertrophied conchal cartilage, underdeveloped antihelical fold, conchoscaphal angle more than $90^{\circ}$ or any combination of the fore-mentioned causes [4-7].

Furnas [5, 6] described his technique where suturing of the conchal cartilage to the mastoid periosteum helps to decrease the conchal prominence and narrows the concho-scaphal angle, but it does not interfere with the condition of the antihelical fold. On the other side, Mustarde [4] described posterior suturing of the auricular cartilage creating a new antihelical fold, but no mentioning of the causes of a prominent ear deformity. Over the years, many surgeons have described a combination of both techniques to manage different factors in a prominent ear deformity with full-thickness trans-cartilaginous multiple incision. The aim was to create the antihelical fold and decrease the conchal hypertrophy with the expected results of weekend auricular cartilage and the possibility of apparent incision sites from the thin skin of the anterior surface of the auricle [10-13].

In 1963, Chong-Chet [9] had described his technique of anterior scoring of the auricular cartilage at the site of the antihelical fold, which showed good results in obtaining a smoother cartilaginous fold. The obstacle with such technique was the thickness of the auricular cartilage in older patients with the possibility of cartilage break.

In this modified anterior scoring technique, a size 4 Diamond burr was used, instead of the original scoring using a scalpel to smooth out and decrease the thickness of the auricular cartilage at the site of the suggested antihelical fold. Such scoring resulted in a more malleable cartilage with less possibility of acute prominence of the antihelical fold postoperatively. In case of a hypertrophied conchal cartilage, scoring of the auricular cartilage

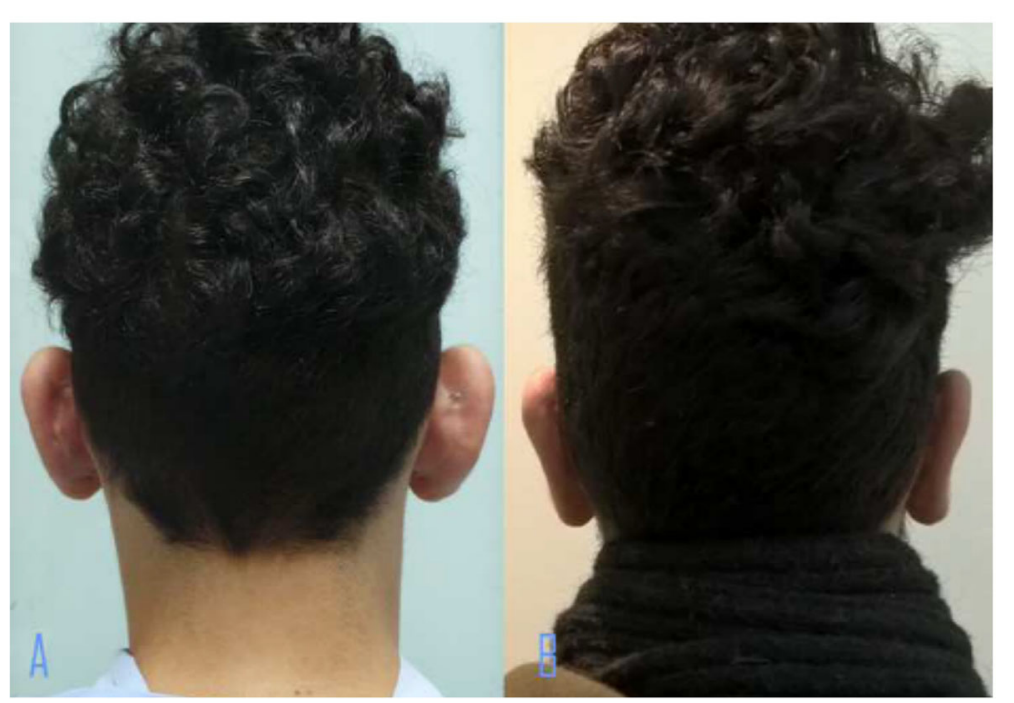

Fig. 8 Preoperative and postoperative pictures of a 27-year-old male patient showing the amount of regression in auricular protrusion 6 months postoperatively 

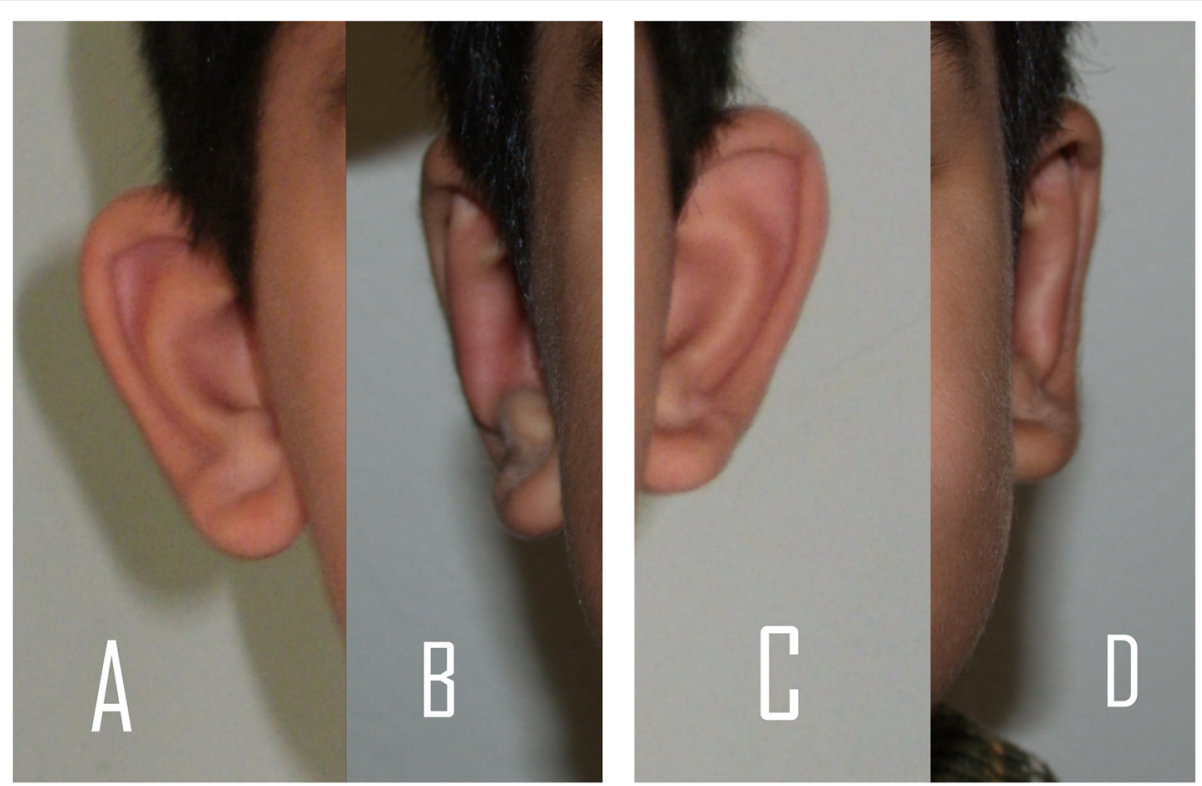

Fig. 9 Preoperative picture of the right and left ears $(\mathbf{a}, \mathbf{c})$ for a 6-year-old child with a bilateral prominent ear deformity with a 1-year postoperative follow-up (b, d)

could be carried out more to the medial side, resulting in a shorter conchal prominence without the need for a separate conchal incision, hence keeping the cartilage integrity.

The author believes that such technique helps in managing all causes of a prominent ear deformity without the need for many incisions in the auricular cartilage, through one incision. Also, this technique could be used in all age groups with no fear of cartilage break in older patient groups. These results were possible to achieve with less relapse of the ear cartilage than that described by Mustarde [4].

\section{Conclusion}

This modification of the anterior scoring technique is very reliable and can manage all the contributing factors causing prominent ears through one technique and through one incision. This technique is suitable for all age groups. It could be used in young children, also, in adults as it alters the rigidity of the auricular cartilage and makes it easier to manipulate.

\section{Acknowledgements}

Not applicable.

\section{Author's contributions}

The author was responsible for the surgical management, data collection, analysis, and writing of the present manuscript. The author(s) read and approved the final manuscript.

\section{Funding}

None.

\section{Availability of data and materials}

The data sets during or analyzed during the current study are available from the corresponding author on reasonable request.

\section{Ethics approval and consent to participate}

All patients have signed a written consent to be included in the present study. In case of children and young patients, parents have signed for the child inclusion and publication in the present study.

The study was approved by the ethical committee of the Faculty of Medicine, University of Alexandria, Egypt (Ethical committee approval and consent to participate ID; 00/19000127).

\section{Consent for publication}

All participating patients have signed an approval for inclusion in the present study and for the usage of their data for publication purposes. In case of children and young patients, parents have signed for the child inclusion and publication in the present study.

\section{Competing interests}

None.

Received: 6 March 2020 Accepted: 6 August 2020

Published online: 18 November 2020

\section{References}

1. Ungarelli et al (2016) Diagnosis and prevalence of prominent lobules in otoplasty: analysis of 120 patients with prominent ears. Aesthet Plast Surg 40(5):645-651

2. Foda H (1999) Otoplasty: a graduated approach. Aesthet Plast Surg 23(6): 407-412

3. McDowell AJ (1968) Goals in otoplasty for protruding ears. Plast Reconstr Surg 41(1):17-27

4. Mustarde JC (1978) Correction of prominent ears using buried mattress sutures. Clin Plast Surg 5(3):459-464

5. Furnas DW (1968) Correction of prominent ears by Concha-Mastoid sutures. Plast Reconstr Surg 42(3):189-193

6. Furnas (1978) Correction of prominent ears with multiple sutures. Clin Plast Surg 5(3):491-495

7. Stenstrom SJ, Heftner J (1978) The Stenstrom otoplast. Clin Plast Surg 5(3): $465-470$ 
8. Stenstrom SJ (1963) A natural technique for correction of congenitally prominent ears. Plast Reconstr Surg 32:509-518

9. Chongchet V (1963) A method of antihelix reconstruction. Br J Plast Surg 16: 268-272

10. Sivrioglu N, Irkoren S, Aksoy B, Copcu E (2012) Chong-Chet anterior scoring technique for the correction of prominent ears: results in 30 patients. Modern Plastic Surg 2:39-42

11. Messner AH, Crysdale WS (1996) Otoplasty: clinical protocol and long-term results. Arch Otolaryngol Head Neck Surg 122(7):773-777

12. Yugueros P, Friedland JA, Furnas DW (2001) Otoplasty: the experience of 100 consecutive patients. Plast Reconstr Surg 108(4):1045-1053

13. Johnson PE (1994) Otoplasty: shaping the antihelix. Aesthet Plast Surg 18(1): 71-74

\section{Publisher's Note}

Springer Nature remains neutral with regard to jurisdictional claims in published maps and institutional affiliations.

\section{Submit your manuscript to a SpringerOpen ${ }^{\circ}$ journal and benefit from:}

- Convenient online submission

- Rigorous peer review

- Open access: articles freely available online

High visibility within the field

- Retaining the copyright to your article

Submit your next manuscript at $\boldsymbol{\nabla}$ springeropen.com 